\title{
Commentary \\ Pulmonary artery occlusion pressure estimation by transesophageal echocardiography: is simpler better?
}

Gorazd Voga

Medical ICU, General Hospital Celje, Oblakova 5, 3000 Celje, Slovenia

Corresponding author: Gorazd Voga, gorazd.voga@guest.arnes.si

Published: 31 March 2008

This article is online at http://ccforum.com/content/12/2/127

(c) 2008 BioMed Central Ltd

See related research by Vignon et al., http://ccforum.com/content/12/1/R18
Critical Care 2008, 12:127 (doi:10.1186/cc6831)

\begin{abstract}
The measurement of pulmonary artery occlusion pressure (PAOP) is important for estimation of left ventricular filling pressure and for distinction between cardiac and non-cardiac etiology of pulmonary edema. Clinical assessment of PAOP, which relies on physical signs of pulmonary congestion, is uncertain. Reliable PAOP measurement can be performed by pulmonary artery catheter, but it is possible also by the use of echocardiography. Several Doppler variables show acceptable correlation with PAOP and can be used for its estimation in cardiac and critically ill patients. Noninvasive PAOP estimation should probably become an integral part of transthoracic and transesophageal echocardiographic evaluation in critically ill patients. However, the limitations of both methods should be taken into consideration, and in specific patients invasive PAOP measurement is still unavoidable, if the exact value of PAOP is needed.
\end{abstract}

Vignon and colleagues [1] prospectively assessed the ability of transesophageal echocardiography (TEE) to predict PAOP higher than $18 \mathrm{mmHg}$ in mechanically ventilated patients with an inserted pulmonary artery catheter. In a first group, they analyzed simple Doppler variables derived from transmitral flow (TMF) and pulmonary venous flow (PVF) and performed the usual measurements and calculations (maximal velocity and velocity time integral of $E$ (the maximal velocity of early diastolic TMF) and $A$ (the maximal velocity of late diastolic TMF) wave, E/A ratio, $E$ wave deceleration time (EDT), maximal velocity and velocity time integral of $S$ (the maximal systolic PVF velocity) and D (the maximal diastolic PVF velocity) wave, $S / D$ ratio, atrial filling fraction and systolic fraction of pulmonary venous flow (SFPVF)). TMF recording was inadequate for analysis in $10 \%$ of patients. The correlations between Doppler variables and pulmonary artery occlusion pressure (PAOP) were better in patients with depressed left ventricular (LV) systolic function than in those with normal LV systolic function. PAOP could be predicted by E/A $>1.4$, EDT $>100 \mathrm{~ms}$, atrial filling fraction $>31 \%$ and SFPVF $>44 \%$, with similar sensitivity and specificity and acceptable positive and negative predictive values. In a second group these cutoff values were prospectively evaluated for prediction of PAOP higher than $18 \mathrm{mmHg}$. Additionally, they measured maximal early diastolic velocity of lateral mitral annulus by tissue Doppler (Ea) and color $\mathrm{M}$-mode Doppler flow propagation velocity (Vp). An E/Ea ratio $<8$ and an E/Vp ratio $<1.7$ were predictive for PAOP $>18 \mathrm{mmHg}$, but the use of these additional variables did not improve the correct estimation of PAOP.

Elevated PAOP reflects an increase of LV end-diastolic pressure due to LV diastolic and/or systolic dysfunction/ failure. PAOP less than $18 \mathrm{mmHg}$, if measured, supports criteria for the definition of acute respiratory distress syndrome and acute lung injury.

Clinical and radiological estimation of PAOP is uncertain in cardiac patients and almost impossible in intensive care unit patients [2-5]. PAOP measurement by pulmonary artery catheter is, for various reasons, not commonly used in cardiac failure and critically ill patients. On the other hand, TEE and transthoracic echocardiography (TTE) are increasingly used for diagnostic and hemodynamic assessment and in critically ill patients, allowing noninvasive estimation of PAOP by Doppler technique [6]. Basically, two groups of Doppler variables are used. The first group includes relatively simple variables (E, A, E/A, EDT, SFPVF) derived from analysis of diastolic TMF and PVF. The second group includes $\mathrm{Ea}$ and $\mathrm{Vp}$; both variables are preload independent and are used to

$A=$ maximal velocity of late diastolic TMF; $D=$ maximal diastolic PVF velocity; $E=$ maximal velocity of early diastolic TMF; Ea $=$ tissue Doppler diastolic velocity of mitral annulus; EDT $=\mathrm{E}$ wave deceleration time; $\mathrm{LV}=$ left ventricular; $\mathrm{PAOP}=$ pulmonary artery occlusion pressure; $\mathrm{PVF}=$ pulmonary venous flow; $\mathrm{S}=$ maximal systolic PVF velocity; SFPVF = systolic fraction of PVF; TEE = transesophageal echocardiography; TMF $=$ transmitral flow; TTE $=$ transthoracic echocardoigraphy; $\mathrm{Vp}=$ color $\mathrm{M}$-mode Doppler flow propagation velocity. 
correct the $E$ velocity for relaxation changes ( $E / E a$ and $E / V p$ ratio).

All variables can be derived by TTE and TEE. In older studies, use of TTE was limited because of inadequate visibility; many patients had to be excluded because of inadequate Doppler signal recordings $[7,8]$. Technical improvements and the use of harmonic imaging now allow measurement of TMF and PVF in the majority of patients, but TEE is still frequently used, especially in mechanically ventilated critically ill patients.

TMF and PVF variables measured by TTE are accurate for the estimation of LV filling pressure and cardiac index in patients with depressed cardiac function and heart failure, but in patients with normal systolic LV function tissue Doppler derived variables show better correlation with PAOP [9-11]. In patients who have undergone cardiac surgery and in critically ill patients, TEE-derived SFPVF and E/Ea correlate well with left atrial pressure and PAOP [12-14].

The study by Vignon and coworkers shows that in patients with acute lung injury, simple Doppler variables derived from TMF and PVF by TEE predicted elevated PAOP better than atrial filling fraction and EDT and that the use of additional and more advanced variables (Ea and $\mathrm{Vp}$ ) did not improve the accuracy of prediction. An important practical limitation of the study is the fact that $20 \%$ of patients could not be studied because of cardiac problems, and that in a further $10 \%$ of patients, some variables could not be recorded.

Concerning the study, the following questions should be considered.

\section{Should we still measure PAOP?}

Despite the fact that PAOP is not transmural pressure and does not accurately reflect preload and volume responsiveness, it is still used as a supportive criterion for the diagnosis of acute respiratory distress syndrome and heart failure. PAOP is, therefore, still measured or estimated in routine clinical practice.

\section{Can we estimate PAOP noninvasively?}

Noninvasive estimation of PAOP is feasible by using TTE/ TEE-derived simple Doppler variables, but not in every patient. Despite technological improvements in past years, adequate Doppler tracing can not be obtained by TTE in many critically ill patients. Also, TEE does not allow adequate recording of Doppler variables in all patients. Additionally, all echo measurements are subjective and require specific operator skill to interpret correctly. It would be interesting to compare TTE and TEE simultaneously for PAOP estimation in a large group of critically ill patients. Besides this, in a certain subset of patients, noninvasive estimation of PAOP is not possible and invasive measurement of PAOP, if needed, is still necessary.

\section{Which variable should we use for noninvasive PAOP estimation?}

Taking into account that TTE or TEE should be performed in the majority of intensive care unit patients for initial hemodynamic assessment, the systematic estimation of PAOP by simple analysis of TMF and PVF would undoubtedly increase the overall quality of this. The use of additional variables (Ea, $\mathrm{Vp}$ ), which are routinely not measured in the intensive care unit setting, is not necessary for PAOP estimation in patients with impaired global systolic LV function, but can improve its estimation in patients with normal systolic function and diastolic dysfunction/failure.

\section{Competing interests}

The author declares that they have no competing interests.

\section{References}

1. Vignon P, AitHssain A, François B, Preux PM, Pichon N, Clavel M, Frat JP, Gastinne H: Echocardiographic assessment of pulmonary artery occlusion pressure in ventilated patients: a transesophageal study. Crit Care 2008, 12:R18.

2. Voga G, Zuran I, Krivec B, Skale R, Pareznik R, Podbregar M: Comparison of clinical and hemodynamic assessment of heart failure in patients with acute myocardial infarction. Zdrav vestn 1997, 66:359-363.

3. Dawson NV, Connors AF Jr, Speroff T, Kemka A, Shaw P, Arkes HR: Hemodynamic assessment in managing the critically ill: is the physician confidence warranted? Med Decis Making 1993, 13:258-266.

4. Herman PG, Khan A, Kallman CE, Rojas KA, Carmody DP, Bodenheimer MM: Limited correlation of left ventricular enddiastolic pressure with radiographic assessment of pulmonary hemodynamics. Radiology 1990, 174:721-724.

5. Staudinger T, Locker GJ, Laczika K, Knapp S, Burgmann H, Wagner A, Weiss K, Zimmerl M, Stoiser B, Frass M: Diagnostic validity of pulmonary artery catheterization for residents at an intensive care unit. J Trauma 1998, 44:902-906.

6. Cholley BP, Vieillard-Baron A, Mebazaa A: Echocardiography in the ICU: time for widespread use! Intensive Care Med 2006, 32:9-10.

7. Nagueh SF, Kopelen HA, Zoghbi WA: Feasibility and accuracy of Doppler echocardiographic estimation of pulmonary artery occlusion pressure in the intensive care unit. $\mathrm{Am} \mathrm{J}$ Cardiol 1995, 75:1 256-1262.

8. Boussuges A, Blanc P, Molenat F, Burnet H, Habib G, Sainty JM: Evaluation of left ventricular filling pressure by transthoracic Doppler echocardiography in the intensive care unit. Crit Care Med 2002, 30:362-367

9. Yamamuro A, Yoshida K, Hozumi T, Akasaka T, Takagi T, Kaji S, Kawamoto T, Yoshikawa J: Noninvasive evaluation of pulmonary capillary wedge pressure in patients with acute myocardial infarction by deceleration time of pulmonary venous flow velocity in diastole. J Am Coll Cardiol 1999, 34:90-94.

10. Masuyama T, Lee JM, Nagano R, Nariyama K, Yamamoto K, Naito J, Mano T, Kondo H, Hori M, Kamada T: Doppler echocardiographic pulmonary venous flow-velocity pattern for assessment of the hemodynamic profile in acute congestive heart failure. Am Heart J 1995, 129:107-113.

11. Rivas-Gotz C, Manolios M, Thohan V, Nagueh S: Impact of left ventricular ejection fraction on estimation of left ventricular filling pressures using tissue Doppler and flow propagation velocity. Am J Cardiol 2003, 91:780-784.

12. Kuecherer HF, Muhiudeen IA, Kusumoto FM, Lee E, Moulinier LE, Cahalan MK, Schiller NB: Estimation of left atrial pressure from transesophageal pulsed Doppler echocardiography of pulmonary venous flow. Circulation 1990, 82:1127-1139.

13. Bouhemad B, Nicolas-Robin A, Benois A, Lemaire S, Goarin JP, Rouby JJ: Echocardiographic Doppler assessment of pulmonary capillary wedge pressure in surgical patients with postoperative circulatory shock and acute lung injury. Anesthesiology 2003, 98:1091-1100 
14. Combes A, Arnoult F, Trouillet JL: Tissue Doppler imaging estimation of pulmonary artery occlusion pressure in ICU patients. Intensive Care Med 2004, 30:75-81. 